\title{
To Study the Relationship between Primary Headache and Attention Deficit in College Going Students - A Correlational Study
}

\author{
Krima Shah ${ }^{1}$ Alpa Purohit ${ }^{2}$ \\ ${ }^{1}$ MPT in Musculoskeletal Conditions, ${ }^{2}$ MPT Musculoskeletal, Vice Principal and Senior Lecturer, \\ Ahmedabad Institute of Medical Sciences, Ahmedabad, Gujarat
}

\begin{abstract}
Aim: To check relationship between primary headache and attention deficit in college going students.

Background: Headache disorders are among the most common disorders of the nervous system. Headache itself is a painful and disabling feature of a small number of primary headache disorders, namely migraine, tension-type headache, and cluster headache.

Attention deficit is a neurological disorder that causes a range of behavior problems such as difficulty attending to instruction, focusing on work, keeping up with assignments, following instructions, completing tasks and social interaction.
\end{abstract}

Due to study and work related load, the subjects are prone to develop headache and this may cause attention problem.

There are very less studies done to correlate headache and attention deficit, so there arises a need to study the relationship between and attention in students and working population.

Methodology: A Study was performed on 143 participants. A structured questionnaires "HEADACHE IMPACT SCALE-6" and MINDFUL ATTENTION AWARENESS SCALE" were filled by the participants and submitted questionnaire through Google forms and personal interview method

Results: 143 participants completed the questionnaires. The value of correlation coefficient is $r=-0.353$, which is statistically significant. Therefore, there is weak negative correlation between headache and attention.

Conclusion: The current study demonstrated that the attention is affected in the college going students having headache. There is a correlation between attention and headache in college going students.

Keywords: Headache, Attention, Headache Impact Scale 6, Mindful Attention Awareness Scale

\section{Corresponding Author:}

Krima Shah

MPT Musculoskeletal, Vice Principal and Senior Lecturer, Ahmedabad Institute of Medical Sciences, Ahmedabad, Gujarat

\section{Introduction}

Headache is a common symptom arising from psychological, ophthalmological, neurological or systemic disease. In clinical practice tension-type headache is encountered most frequently. ${ }^{1}$ Pain or 
discomfort between the orbits and occiput, arising from pain-sensitive structures is called headache. ${ }^{1}$ Headache is one of the commonest symptoms, and primary headache disorders are among the most common disorders, affecting people in all countries. ${ }^{2}$ India appears to be no exception. The Global Burden of Disease Study found tension-type headache (TTH) and migraine to be the 2 nd and 3rd most prevalent disorders worldwide. ${ }^{2}$

Attention deficit disorder (ADD) is a neurological disorder that causes a range of behaviour problems such as difficulty attending to instruction, focusing on work, keeping up with assignments, following instructions, completing tasks and social interaction. ${ }^{2}$ Attention deficit disorder (ADD) and learning disabilities are also common among the students and are considered to be important factors leading to poor academic performance. ${ }^{2}$

\section{Need of the study:}

Concentration difficulties, emotional rigidity, deliberation and hyperactivity as well as stress among the family or at study place are considered to be psychological predictors of headaches in this age group.

Due to headache there are difficulties in focusing on study, decline in the graph of there academic performance. So there arises need to find out the relationship between headache and attention.

There are very less studies done to correlate headache and attention deficit, so there arises a need to study the relationship between and attention in students.

Aim: To study the relationship between primary headache and attention deficit in college going students.

Objective: To correlate headache impact test with the mindful attention awareness scale.

\section{Hypothesis:}

- Null hypothesis: $\mathbf{H}_{\mathbf{0}}{ }^{1}$ : There is no significant correlation between headache impact test and mindful attention awareness scale in college going students.

- Alternate hypothesis: $\mathbf{H}_{\mathbf{1}}{ }^{1}$ : There is significant correlation between headache impact test and mindful attention awareness scale in college going student.

\section{Methodology}

- Source of collection of data : College going students from different colleges of Ahmedabad

- Method of collection of data : The data was collected by interview, mailing, telephonic interview, Google forms.

- $\quad$ Study design - correlational study

- $\quad$ Sample size - 143 subjects

- $\quad$ Age group - 18 to 24 years

- $\quad$ Sampling - convenience sampling

- Materials used :

$$
\begin{aligned}
& \text { - Pen, Paper } \\
& \text { - scales }
\end{aligned}
$$

\section{- Inclusion Criteria}

1. Age: $18-24$ years

2. Males and females

3. Episode of headache within a month

4. Willingness of subject to participate

- Exclusion Criteria

1. Any psychological condition

2. Any other neurological condition

3. History of cognitive impairment

4. Infections

\section{- Outcome Measure:}

. $\quad$ Headache Impact Scale-6( $\alpha-0.92)^{3}$

It consists of 6 questions.

HIT is a tool used to measure the impact headaches have on your ability to function on the job, at school, at 
home and in social situations.

Your score shows you the effect that headaches have on normal daily life and your ability to function.

If your HIT-6 is 50 or higher: You should share your results with your doctor. Headaches that stop you from enjoying the important things in life, like family, work, school or social activities could be migraine.

. The Mindful Attention Awareness $\operatorname{Scale}(\alpha$ $-0.89)^{4}$

The trait MAAS is a 15-item scale designed to assess a core characteristic of mindfulness, namely, a receptive state of mind in which attention, informed by a sensitive awareness of what is occurring in the present, simply observes what is taking place.

Scoring MAAS: To score the scale, simply compute a mean of the 15 items. Higher scores reflect higher levels of dispositional mindfulness.

\section{- Procedure:}

Participants were randomly selected and all met the inclusion and exclusion criteria.

Physiotherapists were asked to fill up the HEIMPACT SCALE 6 and MINDFUL ATTENTION AWARENESS SCALE sent through Google forms and through personal interview.

The questionnaire took approximately 10-15 minutes to complete.

Scores were calculated for each participant.

Data analysis was done using SPSS 16.
All these simple techniques allowed us to assess correlation between headache and attention easily.

\section{Statistical Analysis}

Data was analyzed using SPSS version 16.0.

- The normality of the data was analyzed using the Shapiro-Wilk test.

- Data was not normally distributed, so Spearman's rho test was used.

TABLE 1: GENDER AND AGE DISTRIBUTION

\begin{tabular}{ll}
$\begin{array}{l}\text { DEMOGRAPHIC } \\
\text { DATA }\end{array}$ & $($ MEAN \pm S.D) \\
AGE & $21.41 \pm 1.99$ \\
\hline GENDER & MALE 53 \\
& FEMALE 90 \\
\hline
\end{tabular}

TABLE 2 MEAN DIFFERENCES OF HIT-6 AND MAAS OUTCOME MEASURES (MEAN \pm S.D)
HIT-6
$55.34 \pm 9.55$

MAAS $\quad 4.5 \pm 1.06$

\section{Result}

The value of correlation coefficient is $\mathrm{r}=-0.353$, which is statistically significant.

Therefore, there is weak negative correlation between headache and attention. 
TABLE 3 CORRELATION CHART

\begin{tabular}{|c|c|c|c|c|}
\hline \multicolumn{5}{|l|}{ Correlations } \\
\hline & & & headache & attention \\
\hline \multirow{6}{*}{ Spearman's rho } & \multirow{3}{*}{ headache } & $\begin{array}{l}\text { Correlation } \\
\text { Coefficient }\end{array}$ & 1.000 & $-.353^{* *}$ \\
\hline & & Sig. (2-tailed) & . & .001 \\
\hline & & $\mathrm{N}$ & 143 & 143 \\
\hline & \multirow{3}{*}{ attention } & $\begin{array}{l}\text { Correlation } \\
\text { Coefficient }\end{array}$ & $-.353^{* *}$ & 1.000 \\
\hline & & Sig. (2-tailed) & .001 & \\
\hline & & $\mathrm{N}$ & 143 & 143 \\
\hline
\end{tabular}
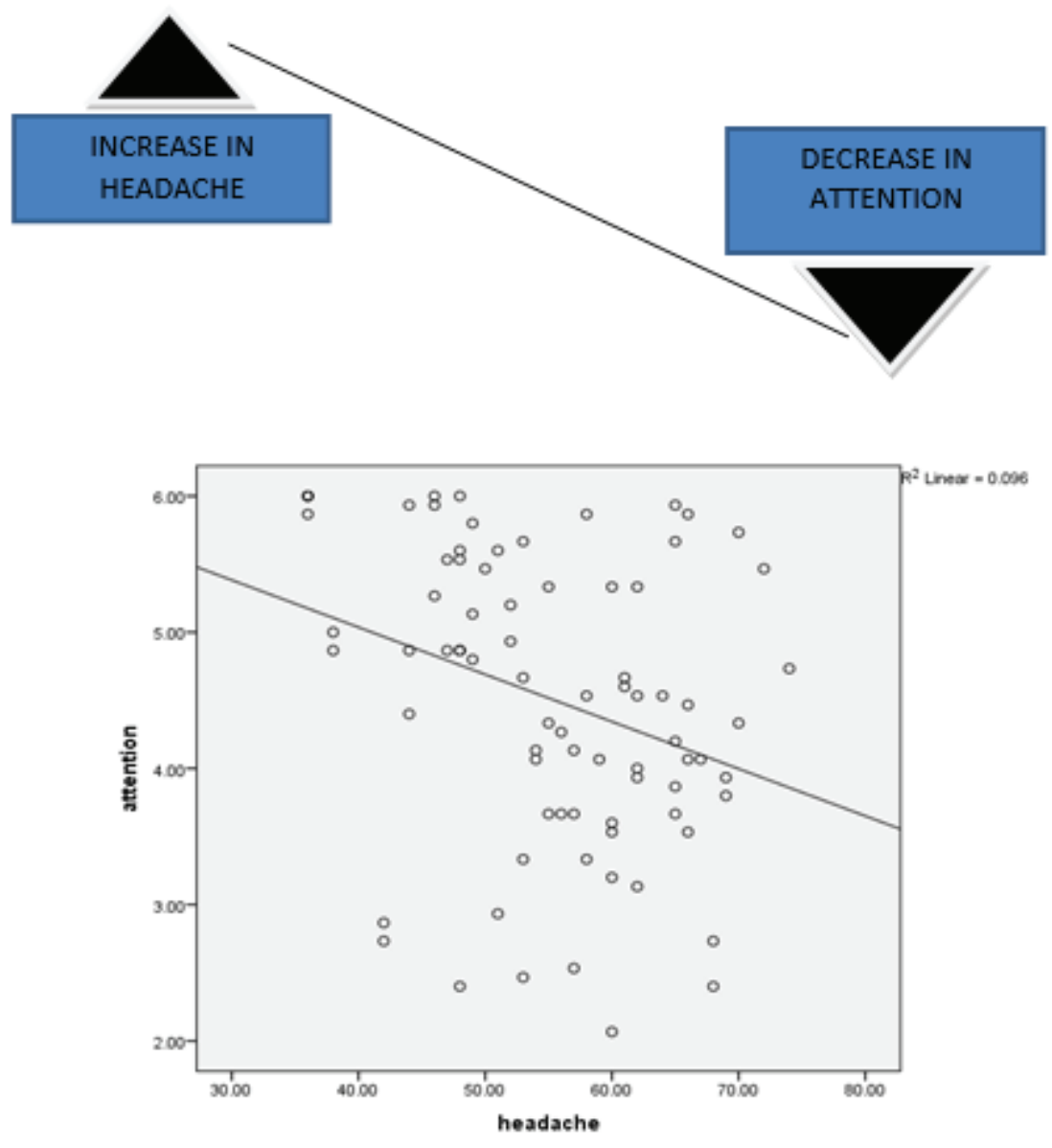

GRAPH 1 INCREASE IN HEADACHE DECREASE IN ATTENTION 


\section{Discussion}

This study is conducted to see the relationship between primary headache and attention in college going students with age group of $18-25$ years.

In this study the result suggests that correlation is found between HIT-6 and MASS scores.

Thus we can define that with increase in headache leads to lack of attention which can affect the academic performance.

Leviton et al was one of the first to report that out of 150 elementary-school children who had recurrent headaches, approximately $40 \%$ had academic difficulties. ${ }^{5}$

Neurotransmitters such as dopamine and noradrenalin are involved in the pathophysiology of migraine; therefore they suggested it may dispose those people to attention deficit. ${ }^{6}$

In recent studies by Riva et al a significant association between attention related problems and headaches was demonstrated in both conditions-migraine and tension type headache. ${ }^{7}$

Some authors suggested that migraine and tension headache form a continuum that may share the same pathophysiological mechanisms and that the cerebral circuits sub serving headache, personality profile, and attention-may overlap ${ }^{8}$

In a population-based study on healthy 4-17 years old children that was conducted by Strine et al, children with frequent headaches were 2.6 times more likely to have inattention .

\section{Conclusion}

The current study demonstrated that the attention is affected in the college going students having headache. There is a correlation between attention and headache in college going students.

\section{Clinical Implication}

. $\quad$ In physiotherapy practice this study can help to give rise to new approaches for headache and attention training.

- Subjects can be assessed for headache in more detailed examination and treatment plan can be made.

. Before treating the symptoms treat the cause which affects the headache.

- Approaches we can use :

- For headache

- Meditation and yoga

- Relaxation exercises

- Cranio vertebral manipulation

- Sub occipital inhibition technique

- Life style modifications

- Aerobic exercise

- For attention

- Attention training

- Behavioural therapy

- $\quad$ Lifestyle modifications

- Meditation and yoga

\section{Limitations and Future Studies}

. $\quad$ Frequency and episodes of headache were not taken.

. In future studies, different population like office workers, students, etc should be taken.

Conflict of Interest: NIL

Source of Funding: Self

Ethical Clearance: Ahmedabad institute of medical sciences 


\section{References}

1) Lindsay KW, Bone I, Fuller G. Neurology and neurosurgery illustrated e-book. Elsevier Health Sciences; 2010 Sep 9.

2) Genizi J, Gordon S, Kerem NC, Srugo I, Shahar E, Ravid S. Primary headaches, attention deficit disorder and learning disabilities in children and adolescents. The journal of headache and pain. 2013 Dec;14(1):1-5.

3) Rendas-Baum R, Yang M, Varon SF, Bloudek LM, DeGryse RE, Kosinski M. Validation of the Headache Impact Test (HIT-6) in patients with chronic migraine. Health and quality of life outcomes. 2014 Dec;12(1):1-0.

4) MacKillop J, Anderson EJ. Further psychometric validation of the mindful attention awareness scale (MAAS). Journal of Psychopathology and Behavioral Assessment. 2007 Dec 1;29(4):289-93.
5) Leviton A. Do learning handicaps and headache cluster?. Journal of child neurology. 1986 Oct;1(4):372-7.

6) Riva D, Aggio F, Vago C, Nichelli F, Andreucci E, Paruta N, D'Arrigo S, Pantaleoni C, Bulgheroni S. Cognitive and behavioural effects of migraine in childhood and adolescence. Cephalalgia. 2006 May;26(5):596-603.

7) Riva D, Usilla A, Aggio F, Vago C, Treccani C, Bulgheroni S. Attention in children and adolescents with headache. Headache: The Journal of Head and Face Pain. 2012 Mar;52(3):374-84

8) Young WB, Peres MF, Rozen TD. Modular headache theory. Cephalalgia. 2001 Oct;21(8):8429.

9) Strine TW, Okoro CA, McGuire LC, Balluz LS. The associations among childhood headaches, emotional and behavioral difficulties, and health care use. Pediatrics. 2006 May 1;117(5):1728-35. 